\title{
Peculiarities of Light Propagation in Photonic Crystal Waveguides in the Slow Light Regime
}

\author{
Lavrinenko, Andrei; Borel, Peter Ingo; Frandsen, Lars Hagedorn; Fage-Pedersen, Jacob
}

Published in:

International Conference on Transparent Optical Networks, 2006

Link to article, DOI:

10.1109/ICTON.2006.248346

Publication date:

2006

Document Version

Publisher's PDF, also known as Version of record

Link back to DTU Orbit

Citation (APA):

Lavrinenko, A., Borel, P. I., Frandsen, L. H., \& Fage-Pedersen, J. (2006). Peculiarities of Light Propagation in Photonic Crystal Waveguides in the Slow Light Regime. In International Conference on Transparent Optical Networks, 2006 IEEE. https://doi.org/10.1109/ICTON.2006.248346

\section{General rights}

Copyright and moral rights for the publications made accessible in the public portal are retained by the authors and/or other copyright owners and it is a condition of accessing publications that users recognise and abide by the legal requirements associated with these rights.

- Users may download and print one copy of any publication from the public portal for the purpose of private study or research.

- You may not further distribute the material or use it for any profit-making activity or commercial gain

- You may freely distribute the URL identifying the publication in the public portal 


\title{
Peculiarities of Light Propagation in Photonic Crystal Waveguides in the Slow Light Regime
}

\author{
Andrei V. Lavrinenko, Peter I. Borel, Lars H. Frandsen, and Jacob Fage-Pedersen \\ COM•DTU, Department of Communications, Optics \& Materials, NanoDTU, Technical University of Denmark, \\ 345v, DK-2800, Kgs. Lyngby, Denmark \\ Tel: (45) 4525 6392,Fax: (45) 4593 6581, e-mail: ala@com.dtu.dk
}

\begin{abstract}
We report on transmission, field mapping, and spectral dependence of the group index in the slow light regime, obtained by numerical modelling. The influence of a finite number of lattice periods on the observed properties is pointed out.
\end{abstract}

Keywords: Photonic crystal waveguides, slow light, transmission, group velocity.

\section{INTRODUCTION}

The area of the current research is the slow-light regime in photonic crystal waveguides (PCWs). This regime is interesting because modes with group velocity significantly smaller than the phase velocity in the bulk material have significantly enhanced light-matter interaction [1]. A number of publications have appeared about slow light applications for devices operating on photonic band gap effects since the first discussions [2-6]. The fact that nonlinear effects are effectively enhanced proportionally to the group index allows for a noticeable shrinkage of the size of photonic components. A lot of efforts are spent now to look for devices with large group indices of the propagating modes. Group velocities several hundreds times lower than the speed of light in vacuum have been reported recently by several groups [7-14]. Thus, the interest in utilizing slow light properties has been rising constantly.

We report in this paper on an investigation of PCW mode properties in the slow mode regime. The PCWs were made by removing one row of holes in the $\Gamma-\mathrm{K}$ direction of a triangular lattice of air holes etched in a dense dielectric like silicon $(n=3.476)$. Mode patterns and transversal and longitudinal energy flux profiles are addressed in Section 2. A closer look at the transmission properties of PCWs near the cutoff of the fundamental mode is made in Section 3. Here the data concerning the group index calculations are placed as well. Conclusions are finally given.

\section{MODE PROFILES}

We start with a comparison of the mode profiles in different parts of the fundamental band. In Figure 1 a typical band diagram for a W1 PCW (hole radius $r=0.3 a$, where $a$ is the lattice constant) is presented together with mode maps for the component $E_{\mathrm{x}}$ of the electric field.
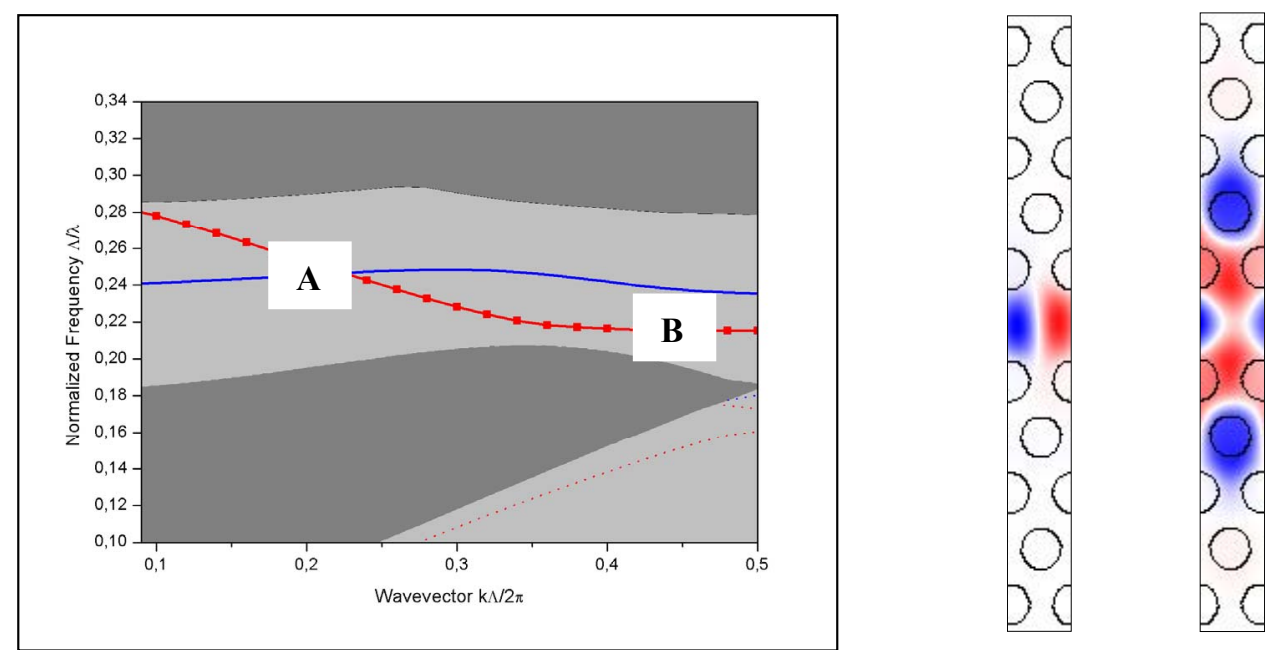

Figure 1. a) TE dispersion diagram for an infinite W1 PCW. The dark grey zones show continuum of photonic crystal modes. b) and c) The transverse profiles of the fundamental mode in points $A$ and $B$, respectively.

The work reported in this paper was supported in part by the Danish Technical Research Council via the PIPE project. 
The diagram was calculated with the 2D plane wave expansion method [15]. The fundamental band gap mode in the linear dispersion regime (point A) is confined within the waveguide core, as can be seen in Fig. $1 b$. However, in the slow-light regime where the dispersion curve is nearly flat (point B) the field spreads out of the core, occupying approximately the two nearest rows of holes on each side of the waveguide.

The mode profiles in the two different parts of a spectrum of a finite W1 PCW were also calculated with the 2D FDTD method. The PCW was $20 \mu \mathrm{m}$ long, having $r=0.3 a$, and $a=475 \mathrm{~nm}$. Results are shown in Fig. 2 for the electric field; the plots agree well with predictions for the infinite periodic structure made by the PWE method. The left-hand side picture is a steady state in the middle of the transmission window, corresponding approximately to point A in Fig. $1(\lambda=2050 \mathrm{~nm})$. The right-hand side picture shows the slow-light mode spreading out from the core $(\lambda=2203 \mathrm{~nm})$, corresponding to point B in Fig. 1. The hole pattern is shown with circles.
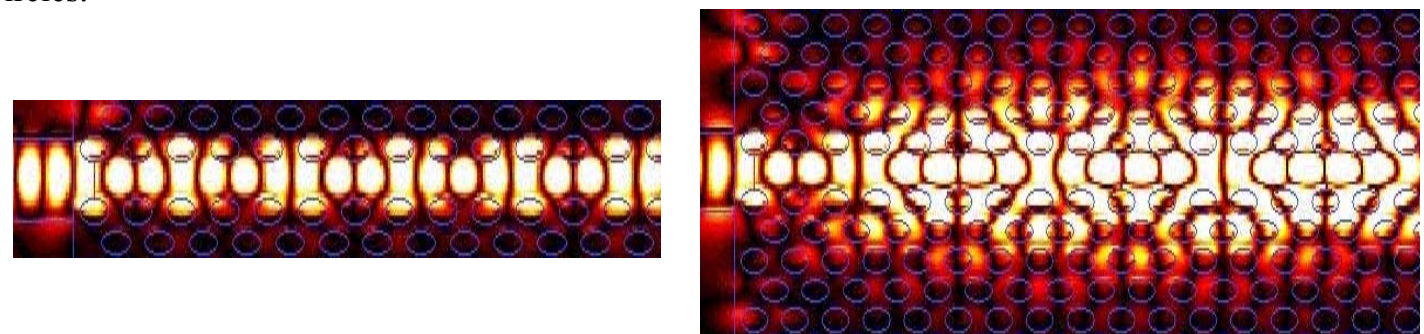

Figure 2. Same as Fig. 1 right, but calculated by 2D FDTD for a 20- $\mu$ m long PCW excited with a continuouswave source. Left picture: in the linear dispersion regime, Right: in the slow mode regime.

To show the mode profiles in more detail, we have plotted the transverse profiles of the field intensity in the PCW in Figure 3. The profiles represent the two wavelengths of 2050 and $2203 \mathrm{~nm}$, corresponding to high transmission and slow light near the cutoff, respectively. The fields were recorded by using 3- $\mu \mathrm{m}$ long transverse oriented detectors, and the zero coordinate is near the centre of the PCW channel.

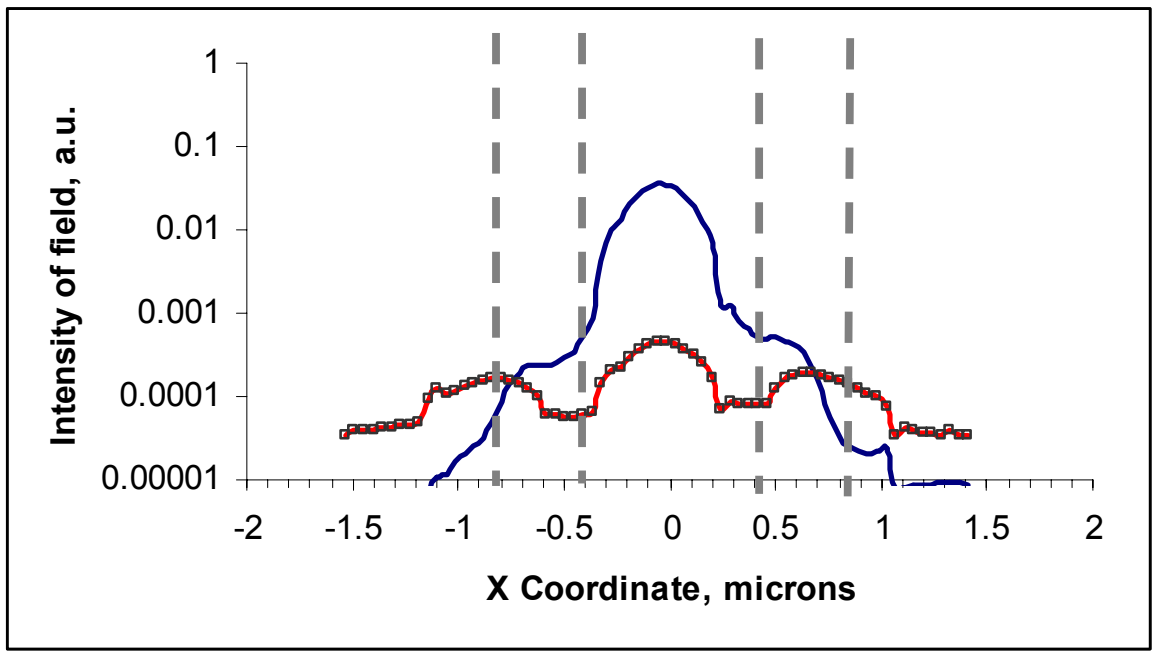

Figure 3. Intensities of the fields in a W1 PCW along a cross section of the core. The solid curve is for $\lambda=2050 \mathrm{~nm}$, the curve with small squares is for $\lambda=2203 \mathrm{~nm}$. The grey dashed lines show the positions of the two rows of holes nearest to the channel of the PCW.

We also placed a detector along the waveguide axis $(z)$ to visualise the longitudinal profile of the mode. The observed intensity profiles are given in Figure 4a. Again, $\lambda=2050 \mathrm{~nm}$ and $2203 \mathrm{~nm}$ are investigated. The intensities were recalculated to be consistent within one graph. The PCW interface is at $z=1 \mu \mathrm{m}$.

We see that there is a transient region inside the PCW after which the fields reach maximum values. The region extends to approximately $1 \mu \mathrm{m}$ from the interface at $\lambda=2050 \mathrm{~nm}$. This distance increases up to $2 \mu \mathrm{m}$ when approaching the cutoff wavelength. Such behaviour resembles to some extent that of field profiles calculated analytically for a $1 \mathrm{D}$ photonic crystal $[16,17]$, although in the cases treated in the references, a wave was used near the photonic band-edge wavelength (no defect modes in the 1D cases).

We analysed the profile of the energy flux. In the first section of the PCW, where the transient region occurs, the net flux in the middle of the channel has only positive values. This can be explained by strong scattering of the fields due to mode mismatch just at the entrance of the PCW, most pronounced for the slow-light mode. Near the peak value of the field, for a short distance the net flux of the slow-light mode becomes dominantly 
negative in the centre of the channel. This is due to accumulation of the fields in the channel after intensive scattering at the entrance. After that, the usual oscillation of the sign of the net flux occurs in the PCW [16, 17]. In the transverse direction, the net flux as a function of $x$ coordinate is shown in Fig. $4 b$. A 3- $\mu$ m long detector had been placed $2 \mu \mathrm{m}$ away from the input interface. In the linear dispersion regime the net flux is mostly confined within the channel (solid curve). In the slow-light regime there are two strong regions of backdraught with negative flux at the positions of the nearest rows of holes.

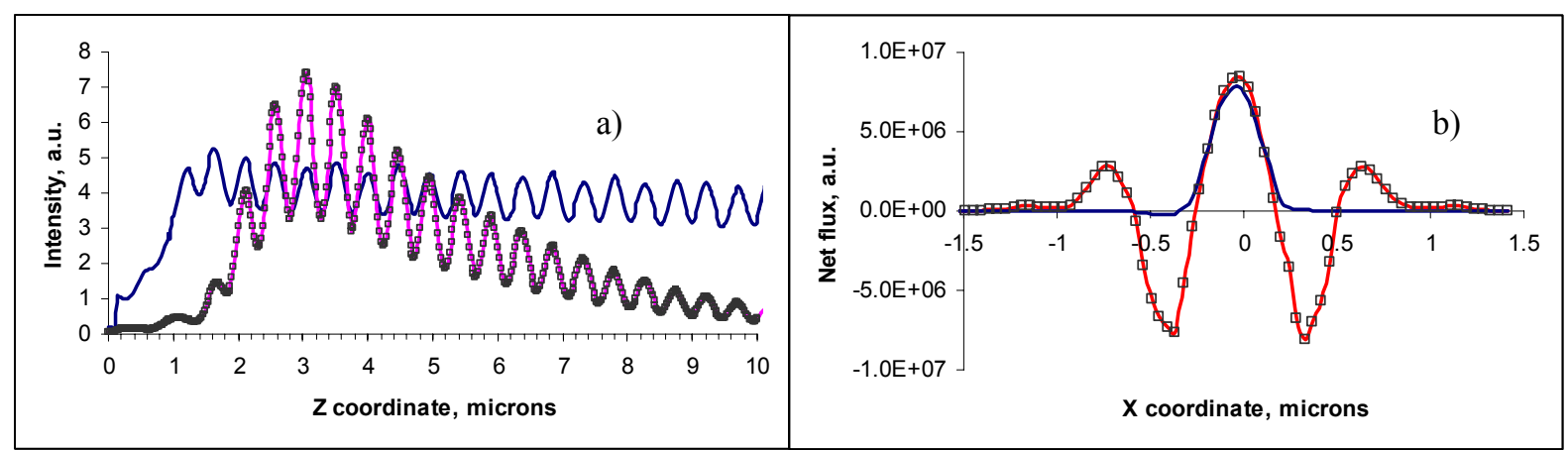

Figure 4. a) Field intensities along the waveguide axis of the W1 PCW, as a function of coordinate. b) Net energy flux inside the PCW, as a function of coordinate along the cross section. In both plots, the solid curve is for $\lambda=2050 \mathrm{~nm}$ and the curve with small squares is for $\lambda=2203 \mathrm{~nm}$.

\section{TRANSMISSION AND GROUP VELOCITY}

An interesting issue is the behaviour of the transmission and group index close to the cutoff frequency. A timeof-flight technique was implemented by direct simulation of pulse propagation in the time domain. The method is described in details in ref. [12], where both 2D and 3D FDTD were employed. The pulse was formed with $1 \cdot 10^{6}$ time steps, providing a FWHM of approximately $0.36 \mathrm{~nm}$. However, fast oscillations of the spectral curves that were observed in experiments called for a higher frequency resolution in the simulations. The simulations were repeated in $2 \mathrm{D}$ with pulses of $4 \cdot 10^{6}$ time steps. Such wide time envelope gives a $0.09-\mathrm{nm}$ resolution in the frequency domain. This was sufficient to see sharp in-phase oscillations of transmission and group index. Results of the modelling are presented in Figure 5. Similar, distinct oscillations of the group index in experiments have been observed recently $[12,13]$. The period of oscillations decreases monotonically when approaching the cutoff wavelength. The number of oscillations and their period is determined by the number of longitudinal lattice periods and is a signiture of the finite length of the PCW. In the 1D case the same effect was investigated in ref. [17].

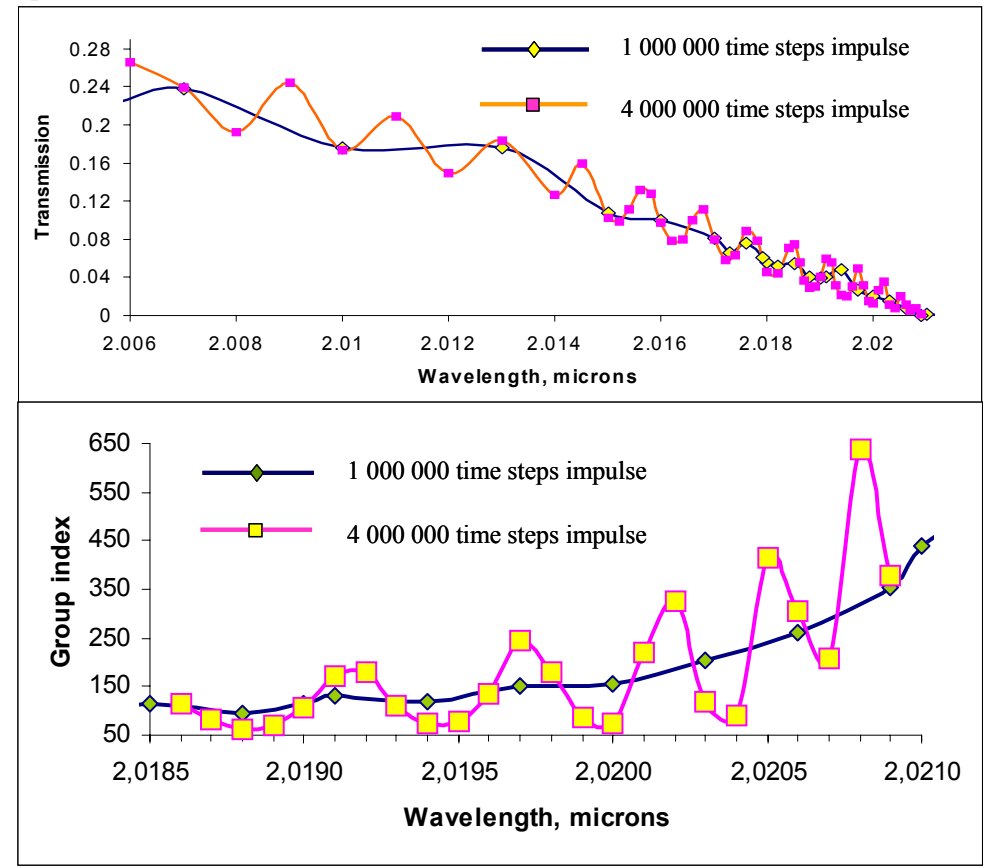

Figure 5. Transmission spectra (upper plot) and group index dependence on the wavelength (lower plot) close to the cutoff of a W1 PCW. The parameters of the waveguide are the same as in Ref.12. 


\section{CONCLUSIONS}

We analysed the properties of guided modes in PCWs in the slow-light regime. The fields profiles show significant spreading of the fields out of the waveguide channel. This fact can be exploited to design structures with deliberate engineering of the slow-light behaviour, e.g. by changing parameters in the second nearest row of holes only. In this way, the properties of the modes propagating in the linear dispersion regime will remain unaffected. Field patterns, intensity profiles in the transverse and longitudinal directions, and diagrams of the net flux show significant differences for the slow-light behaviour in comparison with the ordinary linear dispersion regime. We found, e.g., the existence of zones with large negative net flux in the slow-light regime.

The spectral dependencies of the transmission and group index were calculated in the proximity of the fundamental mode cutoff. To observe rapid oscillations of waveguide characteristics in wavelengths, the frequency resolution of the time-of-flight method was increased four-fold. In analogy with the properties of 1D periodic stacks, the conclusion is made that the observed properties are dominantly governed by the finite length of the periodic lattice.

\section{REFERENCES}

[1] M. Soljacic, et al.: Photonic-crystals slow-light enhancement of nonlinear phase sensitivity, J. Opt. Soc. Amer. B, vol.19, pp. 2052-2059, 2002.

[2] M. Soljacic and J. D.Joannopoulos: Enhancement of nonlinear effects using photonic crystals, Nature materials, vol. 3, pp. 211-219, 2004.

[3] S. Inoue and Y. Aoyagi: Design and fabrication of two-dimensional photonic crystals with predetermined nonlinear optical properties, Phys. Rev. Lett., vol. 94. pp. 103904, 2005.

[4] C. Sauvan, P. Lalanne, and J. P. Hugonin: Slow-wave effect and mode-profile matching in photonic crystal microcavities, Phys. Rev. B, vol. 71, pp. 1651118, 2005.

[5] Y. Chen and S. Blair: Nonlinearity enhanced in finite coupled-resonator slow-light waveguides, Opt. Express, vol.12, pp. 3353-3366, 2004.

[6] J. Hwang, et al.: Enhanced nonlinearity near the photonic band edges of a cholesteric liquid crystal, Opt. Lett. vol. 29, pp. 2644-2646, 2004.

[7] M. Notomi, et al:: Extremely large group-velocity dispersion of line-defect waveguides in photonic crystal slabs, Phys. Rev. Lett., vol 87 pp 253902, 2001.

[8] D. Coquilat, et al:: Observation of band structure and reduced group velocity in epitaxial GaN-sapphire 2D photonic crystals, Appl. Phys. B, vol. 73, pp. 591-593, 2001.

[9] K. Inoue, et al.: Observation of small group velocity in two-dimensional AlGaAs-based photonic crystal slabs, Phys. Rev. B, vol. 65, pp. 121308, 2002.

[10] T. Asano, et al.: Time-domain measurement of picosecond light-pulse propagation in a two-dimensional photonic crystal-slab waveguide, Appl. Phys. Lett., vol. 8 ,pp. 4690-4692, 2004.

[11] H. Gersen, et al.: Real-space observation of ultraslow light in photonic crystal waveguides, Phys. Rev. Lett. vol. 94, pp. 073903, 2005.

[12] R.S. Jacobsen, et al:: Direct experimental and numerical determination of extremely high group indices in photonic crystal waveguides, Opt. Express, vol. 13, pp. 7861-7871, 2005.

[13] Y.A. Vlasov, et al.: Active control of slow light on a chip with photonic crystal waveguides, Nature, vol.438, pp. 65-69, 2005.

[14] R.J.P. Engelen, et al.: The effect of higher-order dispersion on slow light propagation in photonic crystal waveguides, Opt. Express, vol. 14, pp. 1658-1672, 2006.

[15] S. G. Johnson and J. D. Joannopoulos, Block-iterative frequency-domain methods for Maxwell's equations in a planewave basis, Opt. Express, vol. 8, pp. 173-190, 2001.

[16] J. P. Dowling, et al.: The photonic band edge laser: A new approach to gain enhancement, J.Appl. Phys., vol.75, pp. 1896-1899, 1994.

[17] M. Scalora, et al.: Ultrashort pulse propagation at the photonic band edge: Large tunable group delay with minimal distortion and loss, Phys.Rev. E, vol.55, pp. R1078-R1081, 1996. 